\section{Leishmaniose visceral na cidade do Rio de Janeiro - Brasil}

* Instituto Oswaldo Cruz - FIOCRUZ, Rio de Janeiro

* Escola Nacional de Saúde Pública - FIOCRUZ, Rio de Janeiro

** Secretaria Municipal de Saúde do Rio de Janeiro - SMSRJ

*** * Universidade Federal do Rio de Janeiro - UFRJ

***** Superintendência das Campanhas do Ministério da Saúde - SUCAM-RJ

***** Universidade Federal Rural do Rio de Janeiro - UFRRJ

A leishmaniose visceral (LV) ou Calazar Neotropical, no Brasil, é uma zooantroponose peri-urbana e rural. No âmbito doméstico, o cão é importante reservatório do agente, a Leishmania donovani chagasi, e responsável pelo caráter endêmico-epidêmico da doença. No ambiente silvestre, a raposa Lycalopex vetulus ${ }^{3}$ e outros canídeos como o Cerdocion thous ${ }^{6}$ são incriminados como hospedeiros naturais. A única espécie de flebótomo incriminada na transmissão é a Lutzomyia longipalpis, cuja distribuição se superpoe às áreas endêmicas ${ }^{3}$. Clinicamente, a doença humana pouco difere das ou tras modalidades clínico-epidemiológicas existentes em outros continentes, incluindo febre, anemia, hepatoesplenomegalia e emagrecimento de caráter progressivo, porém muito sensível ao tratamento pelo antimonial. A taxa de mortalidade dos casos não tratados varia de 75 a $95 \%$, admitindo-se porém a ocorrência de formas oligossintomáticas e assintomáticas ${ }^{23}$. Segundo o Ministério da Saúde SUCAM, mais de mil casos humanos vêm ocorrendo anualmente no Brasil. Sua distribuição geográfica vai desde o norte da Amazônia até os estados da região sudeste, passando pelos da região centro-oeste. São mais atingidos os estados da região nordeste ( $97 \%$ dos casos), predominando no Ceará. 
Recentemente, além da recrudescência observada nas áreas endêmicas do nordeste, novos focos têm sido detectados no sul do país (Paraná, 3 casos; São Paulo, 2 casos; Rio de Janeiro, 35 casos), sugerindo uma expansão da endemia ${ }^{11}$.

O calazar humano endêmico é mais freqüente em crianças, na maioria das áreas, correspondendo a menores de 10 anos $80 \%$ dos casos, sendo que $60 \%$ ocorrem até os 4 anos; é também predominante no sexo masculino ${ }^{1}$.

O calazar canino enzoótico é mais prevalente que o humano e precede a endemia ${ }^{1}$. É de evolução lenta, levando a acentuado emagrecimento, perda de pelos e apatia, porém freqüentemente assintomático, embora com alto grau de parasitismo na pele sã e vísceras ${ }^{3}$.

A L. longipalpis coloniza facilmente o peridomicílio, adaptando-se a temperaturas elevadas e baixo grau de umidade, sendo o período de maior transmissibilidade a estação chuvosa; os insetos invadem o domicílio, picando tanto o homem quanto o cão ${ }^{3}$.

$\mathrm{O}$ aumento verificado em sua distribuição para o sul do país, em áreas onde sua presença não havia sido notada, justificaria o aparecimento de novos focos da doença $\mathrm{a}^{22}$.

O calazar humano, nunca antes referido na cidade do Rio de Janeiro, foi detectado ali, pela primeira vez, em $1977^{21}$. Até outubro de 1983, 35 casos autóctones foram diagnosticados em áreas peri-urbanas da cidade, situadas nas vertentes continentais norte (bairros de Realengo e Bangu), noroeste (Senador Camará), oeste (Campo Grande) e sudeste (Jacarepaguá) do Maciço da Pedra Branca (XVII, XVIII e XXIV RAs) - Mapa e Fig. 1.





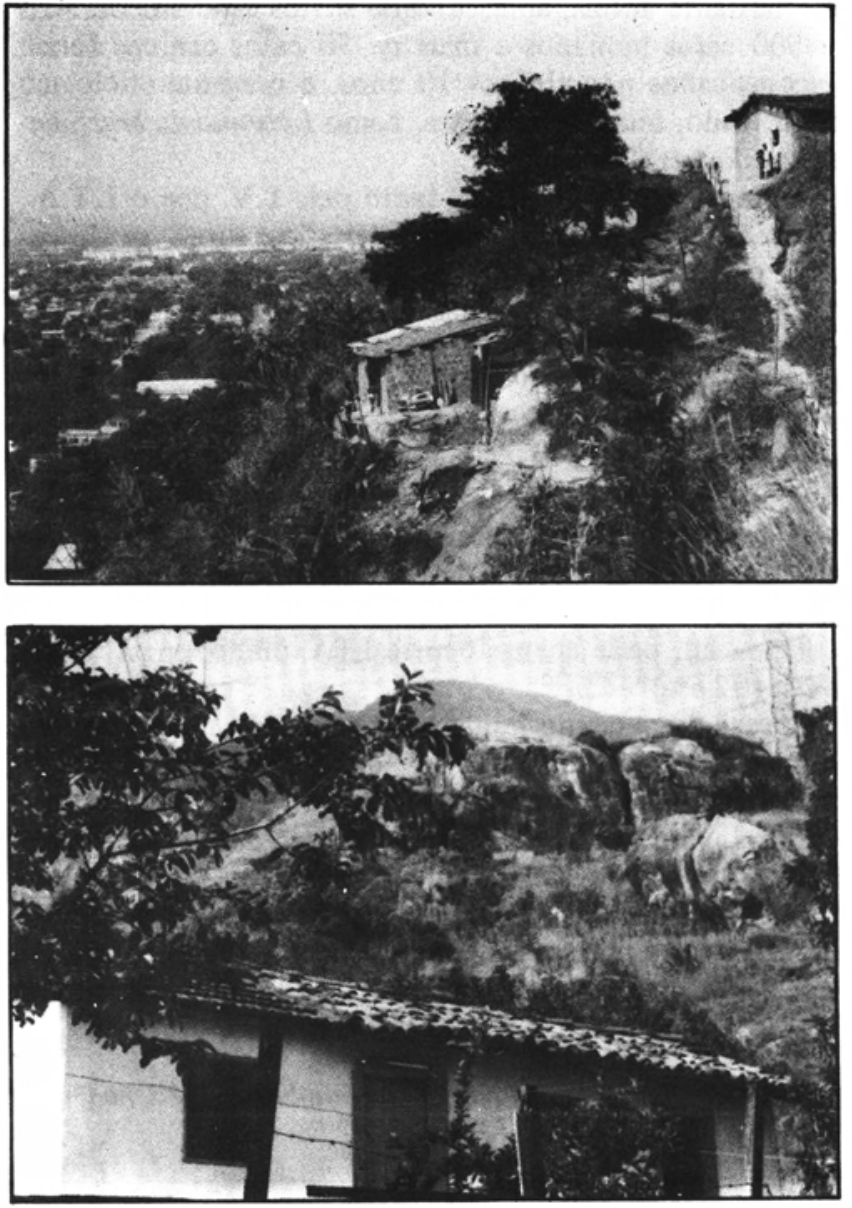

O maciço da Pedra Branca, contraforte da Serra do Mar, situado no Centro do Município, entre os paralelos 22050' e 23005' e os meridianos 43035' e 43020', é um conjunto montanhoso formado por rochas graníticas e gnaíssicas, o mais alto do município, cujo ponto culminante é o Pico da Pedra Branca $(1.024 \mathrm{~m})$. O clima predominante nas vertentes norte e noroeste, de maior insolação, é do tipo Aw, com temperatura média anual de $27,7{ }^{\circ} \mathrm{C}$; a precipitação pluviométrica total anual é maior que $1.400 \mathrm{~mm}$, com menos de $60 \mathrm{~mm}$ nos meses mais secos (julho e agosto) e a umidade relativa pouco variável, em torno de $75^{\circ} \mathrm{C}$. Nas vertentes atlânticas, onde a umidade proveniente do mar encontra no relevo um obstáculo, acarretando chuvas constantes no sopé, o clima predominante nas baixadas é do tipo Am, mais úmido, e Af, chuvoso, nas encostas ${ }^{19}$.

Esse maciço, que parece funcionar como uma barreira ecológica, tem suas vertentes atlânticas voltadas para o bairro de Jacarepaguá (XVI e XXIV Regiões Administrativas), onde a leishmaniose tegumentar americana (LTA) é endêmi-
Fig. 1 - Vista parcial de áreas de ocorrência de calazar (Realengo e Bangu), Rio de Janeiro. 
ca e bastante antiga, apresentando surtos esporádicos. Mais de 500 casos humanos e mais de 50 casos caninos foram diagnosticados nos últimos 10 anos, e o agente etiológico identificado, bioquimicamente, como Leishmania braziliensis braziliensis ${ }^{10}$.

As regiбes mais atingidas, tanto pela L.V. como L.T.A., são áreas peri-urbanas, com características rurais, geralmente de encostas de morros; a população predominante é de baixa renda, e as habitaçðes são precárias e sem equipamen. tos sanitários ou iluminação elétrica, porém fixada à terra há várias décadas.

A fauna flebotomínica, na vertente norte, é constituída por um predominio de $L$. longipalpis $(97,9 \%)$ nas cotas de altitu de acima de $100 \mathrm{~m}$, en quanto, abaixo de $100 \mathrm{~m}$, predomina $L$. intermedia; na vertente oeste, onde são também referidos casos de LTA, a $L$. intermedia assume proporção de $82,4 \%$, enquanto $L$. longipalpis é de $4,6 \%$, e na vertente litorânea sul, onde apenas ocorre LTA, predomina $L$. intermedia $(92,6 \%)^{6}, 20,22$.

Apesar do pequeno número de casos de calazar humano no Rio de Janeiro, o coeficiente de morbidade nas áreas de risco chegou a 28,1 por 10.000 hab. em 1981 (CampoGrande), declinando com as medidas de controle adotadas pela SUCAM (busca ativa e tratamento dos casos humanos, eliminação dos cães infectados e dedetização dos domicílios) e apresentando uma morbidade média geral, no período de 1977 a 1983 , de 4,4 por 10.000 hab. (Tabela I). A distribui-

TABELA I

Morbidade *Anual por Leishmaniose Visceral, no perlodo de agosto de 1977 a outubro de 1983, no munictpio do Rio de Janeiro, $R J$

\begin{tabular}{|c|c|c|c|c|c|c|c|}
\hline \multirow{2}{*}{ Áreas } & \multirow{2}{*}{$\begin{array}{l}\text { População } \\
\text { de Risco }\end{array}$} & \multicolumn{2}{|c|}{1977} & \multicolumn{2}{|c|}{1978} & \multicolumn{2}{|c|}{1979} \\
\hline & & Casos & (Morb) & Casos & (Morb) & Casos & (Morb) \\
\hline $\begin{array}{l}\text { BANGU } \\
\text { (Serra do Bangu) }\end{array}$ & 4316 & 1 & $(2,32)$ & - & - & 1 & $(2,32)$ \\
\hline $\begin{array}{l}\text { REALENGO } \\
\text { (Serra do Barata) }\end{array}$ & 4469 & - & - & - & - & 2 & $(4,48)$ \\
\hline $\begin{array}{l}\text { CAMPO GRANDE } \\
\text { (Rio da Prata e } \\
\text { Carapiá) }\end{array}$ & 2492 & - & - & - & - & 2 & $(8,03)$ \\
\hline $\begin{array}{l}\text { JACAREPAGUÁ } \\
\text { (Rio Bonito) }\end{array}$ & 246 & - & - & - & - & - & - \\
\hline Total & 11523 & 1 & $(0,87)$ & - & - & 5 & $(4,34)$ \\
\hline
\end{tabular}

(*) Coef. morb. por 10.000 habs.

(**) Início das medidas de controle pela SUCAM 
ção etária nas localidades situadas nas vertentes norte (exclusiva de LV) - 19 casos - correspondeu a 39,3\% até 9 anos de idade e $60,7 \%$ acima dessa faixa; nas vertentes oeste, (onde também ocorre LTA) - 15 casos - foi de $66,7 \%$ abaixo de 9 anos e 33,3\% acima de 10 anos. No geral, houve predomínio do sexo masculino (60\%). A ocorrência de apenas $25,7 \%$ dos casos abaixo de 4 anos e $45,7 \%$ acima de 10 anos reforça a possibilidade de implantação recente da endemia (Tabela II).

De 22 doentes por nós examinados, em 21 (95,5\%) evidenciamos Leishmania em material de mielopunção e em 13 (59\%) isolamos o parasito em meio de cultivo ${ }^{12}$.

Todos apresentaram sorologia positiva à imunofluorescência indireta (IF) nas classes IgG, cujos títulos variaram de 1: 90 a 1:2800, com tendência a manterem-se elevados por mais de 6 meses $^{11}$. Na classe IgM foram observados $28,5 \%$ de resultados falsos positivos, relacionados ao fator reumatóide, embora $35,7 \%$ apresentassem anticorpos IgM específicos $^{2}$.

Além da evidenciação de altos níveis de imunocomplexos (IC) e ocorrência de ativação policlonal, observou-se estreita associação entre IC e auto-anticorpos antimúsculo liso $^{4}$. No estudo da patogênese da anemia, Pontes de Carvalho et alii ${ }^{17}$ sugeriram a participação de auto-anticorpos eritrocitários ou da adsorção passiva de imunocomplexos pelos eritrócitos.

\begin{tabular}{|c|c|c|c|c|c|c|c|c|c|}
\hline \multicolumn{2}{|c|}{$1980 * *$} & \multicolumn{2}{|c|}{1981} & \multicolumn{2}{|c|}{1982} & \multicolumn{2}{|c|}{1983} & \multicolumn{2}{|c|}{ Total } \\
\hline Casos & (Morb) & Casos & (Morb) & Casos & (Morb) & Casos & (Morb) & Casos & $\begin{array}{l}\text { (Morb. } \\
\text { Média) }\end{array}$ \\
\hline - & - & 3 & $(6,95)$ & 1 & $(2,32)$ & 1 & $(2,32)$ & 7 & $(2,32)$ \\
\hline 9 & $(20,14)$ & 1 & $(2,24)$ & - & - & - & - & 12 & $(3,84)$ \\
\hline - & - & 7 & $(28,09)$ & 6 & $(24,08)$ & - & - & 15 & $(8,60)$ \\
\hline - & - & - & - & - & - & 1 & $(40,65)$ & 1 & $(5,81)$ \\
\hline 9 & $(7,81)$ & 11 & $(9,55)$ & 7 & $(6,07)$ & 2 & $(1,74)$ & 35 & $(4,40)$ \\
\hline
\end{tabular}


TABELA II

Distribuição por sexo e grupo etário dos casos de Leishmaniose Visceral ocorridos no municlpio do Rio de Janeiro (1977 - 1983)

\begin{tabular}{|c|c|c|c|c|c|c|}
\hline \multirow{3}{*}{$\begin{array}{l}\text { Grupo } \\
\text { etário } \\
\text { (anos) }\end{array}$} & \multicolumn{4}{|c|}{ Sexo } & \multirow{2}{*}{\multicolumn{2}{|c|}{ Total }} \\
\hline & \multicolumn{2}{|c|}{ Masc } & \multicolumn{2}{|c|}{ Fem } & & \\
\hline & No & (\%) & No & (\%) & № & (\%) \\
\hline $\begin{array}{c}0-4 \\
5-9 \\
10-14 \\
15-19 \\
20-39 \\
\geqslant 40\end{array}$ & $\begin{array}{l}2 \\
4 \\
1 \\
5 \\
5 \\
4\end{array}$ & $\begin{array}{r}(5,7) \\
(11,4) \\
(2,9) \\
(14,3) \\
(14,3) \\
(11,4)\end{array}$ & $\begin{array}{l}7 \\
6 \\
- \\
- \\
1 \\
-\end{array}$ & $\begin{array}{r}(20,0) \\
(17,1) \\
(-) \\
(-) \\
(2,9) \\
(-)\end{array}$ & $\begin{array}{r}9 \\
10 \\
1 \\
5 \\
6 \\
4\end{array}$ & $\begin{array}{r}(25,7) \\
(28,6) \\
(2,9) \\
(14,3) \\
(17,1) \\
(11,4)\end{array}$ \\
\hline Total & 21 & $(60,0)$ & 14 & $(40,0)$ & 35 & $(100,0)$ \\
\hline
\end{tabular}

A intradermorreação de Montenegro (IDRM), realizada antes do tratamento, foi, surpreendentemente, positiva em um paciente de 19 anos, procedente da encosta norte; após o tratamento, a IDRM foi positiva em 50\% dos indivíduos testados, com tendência a permanecer negativa nos indivíduos mais jovens ${ }^{12}$.

O tempo referido da doença variou de 10 dias a 5 meses; hepatoesplenomegalia e anemia, presentes em todos os casos, foram de intensidade variável, sendo que febre, durante a internação, deixou de ocorrer em apenas um caso que apresentou icterícia à admissão; as al teraçðes laboratoriais clássicas puderam ser observadas na maioria dos $\operatorname{casos}^{24}$.

Cerca de $25 \%$ dos pacientes apresentaram alterações hemorrágicas de pele, antes ou durante o tratamento específico; nestes, a plaquetopenia, observada à internação ou concomitante ao agravamento do quadro hemorrágico, associouse à acentuada elevação de imunocomplexos circulantes ${ }^{8}$.

Quatro de seis casos (com idades de 22 a 36 anos), reavaliados clinicamente até um ano após o diagnóstico, apresentaram hipertensão arterial persistente; desses, três tiveram a forma sintomática e foram tratados após comprovação diagnóstica, e um, oligossintomático, apenas com IF e IDRM positivas, não foi tratado; porém, três deles eram irmãos (um dos quais oligossintomático), com antecedentes familiares de hipertensão ${ }^{9}$.

Ao tratamento específico feito pela administração endovenosa de Glucantime (Rhodia-CEME), na dose de 50-100 $\mathrm{mg} / \mathrm{kg} / \mathrm{dia}$, em dois períodos de 10 dias, com 10 dias de intervalo, não se observou resistência à droga. Os 3 óbitos ocorridos foram associados ao início tardio da terapêutica em pacientes com estado geral muito comprometido e agravado por complicaçð̃es (sépsis e hemorragia digestiva).

Um inquérito sorológico canino pela reação de imunofluorescência indireta, realizado pela SUCAM com colaboração da FIOCRUZ, evidenciou em cães de áreas exclusiva- 
mente com LV (Realengo, Bangu e Senador Camará) uma prevalência de $4,3 \%$ de positividade; em cães da área mista de LV e LTA (Campo Grande), uma prevalência de 12,7\% e em cães de Pau da Fome (Jacarepaguá), na vertente litorânea sudeste do Maciço da Pedra Branca, onde têm ocorrido apenas casos humanos e caninos de leishmaniose tegumentar, $8,6 \%$. $^{*}$

Em 40 cães recolhidos das áreas endêmicas, com diagnóstico parasitológico ou sorológico positivos, observamos que, em 19 cães das áreas exclusivas de LV, apenas 36,8\% apresentavam sinais clínicos sugestivos da infecção (emagrecimento - 100\%; infartamento ganglionar e depilação - 85,7\%; apatia - 71,4\%; ulceraçðes, descamações furfuráceas e unhas alongadas - 42,9\%); em 21 cães provenientes da área mista de LV e LTA, 76,2\% apresentavam sinais sugestivos, predominando, porém, as ulceraçóes de pele ou mucosas; outros sinais menos freqüentes, como evacuações sanguinolentas, queratoconjuntivite e paresia, foram observados em ambas as áreas; o exame parasitológico, após necrópsia dos 40 cães, evidenciou Leishmania em 39 , sendo $42,5 \%$ em vísceras e pele normal, $35 \%$ somente em ulceraçбes de pele ou mucosas e $20 \%$ somente em vísceras; todas as amostras de Leishmania isoladas da área de Realengo (LV) foram caracterizadas bioquimicamente (isoenzimas, anticorpos monoclonais e kDNA) como L. donovani chagasi; nas amostras isoladas da área de Campo Grande (LV + LTA) e caracterizadas pelos mesmos métodos, observou-se tanto L.d. chagasi como L.b. braziliensis; o tratamento com antimonial (Glucantime) de 3 cães positivos aos exames parasitológicos e sorológicos, porém apresentando regular estado geral, não logrou êxito, observando-se, porém, agravamento das manifestações clínicas e morte precoce de um animal. *

Um inquérito epidemiológico através de exame clínico, IF e IDRM, realizado em 121 moradores da encosta norte (Serra do Barata - Realengo), excluindo-se os 11 individuos que tiveram LV na mesma localidade, evidenciou um caso sintomático, com confirmação parasitológica posterior e um caso assintomático, com IF e IDRM positivas e sem confirmação parasitológica, além de três indivíduos $-2,5 \%$ (dois adultos e uma criança) com apenas IDRM positiva ${ }^{13}$.

$\mathrm{Na}$ encosta oeste (Rio da Prata - Campo Grande), Toledo et alii $^{25}$ aplicaram o mesmo tipo de inquérito a duas subáreas distintas, numa (170 individuos) predominando LV e noutra (213 indivíduos) predominando LTA; na subárea de LV, a prevalência da IDRM (12\%) foi constante em todas as faixas etárias e variou de $9,4 \%$ a $18,9 \%$, enquanto na subárea de LTA a prevalência da IDRM (29\%) mostrou comportamento crescente em direção aos grupos etários mais altos, variando de $5,4 \%$ a $50 \%$.

$\mathrm{Na}$ subárea de $\mathrm{LV}, 75$ moradores foram divididos em quatro grupos, de acordo com a ocorrência ou não de casos
* COUTINHO, S.G. et alii. A survey of visceral and cutaneous leishmaniasis among 1.342 dogs from areas in Rio de Janeiro (Brazil) where the human disease occurs. Trabalho aceito para publicação nas Mem. Inst. Oswaldo Cruz.

** MARZOCHI, M.C.A. et alii. Leishmaniose visceral canina no Rio de Janeiro, Brasil. Aspectos clínico-parasitológicos $e$ epidemiológicos observados (1977.1983). Trabalho em preparo. 
humanos e caninos em suas residências ${ }^{25}$. No Grupo I, onde ocorreram casos humanos, a IF foi de zero por cento e a IDRM de 10\%, no Grupo II, onde ocorreram apenas casos caninos, a IF foi de 10,3\% e a IDRM de 13,8\%; no Grupo III, onde ocorreram casos humanos e caninos, a IF foi de $15,4 \%$ e a IDRM de $23,1 \%$ e, no Grupo IV, onde não ocorreram casos humanos ou caninos, a IF foi de zero por cento e a IDRM de $3,9 \%$. A presença de cinco indivíduos $(6,6 \%$ da população testada) dos grupos II e III clinicamente normais, mas positivos à IF e à IDRM, sugeriu a ocorrência de formas subclinicas ou oligossintomáticas; o cultivo de mononucleares, separados do sangue periférico em gradiente de FicollHypaque, de três desses indivíduos, mostrou a presença de formas promastigotas de Leishmania; no entanto, não se encontrou Leishmania em material de medula óssea, tanto no esfregaço corado como no cultivo em meio $\mathrm{NNN}^{25}$

A proximidade da população da encosta oeste com área onde ocorre a LTA humana e canina, ao contrário do observado na encosta norte, poderia justificar a maior prevalência da IDRM positiva e talvez explicar a menor ocorrência de LV em adultos, sugerindo uma proteção imunológica cruzada dos grupos etários mais velhos pela L.b. braziliensis, uma vez que a LTA parece ser de implantação mais antiga e a LV mais recente.

Por outro lado, as formas frustras ou assintomáticas não parecem ser raras e, provavelmente, desempenhem papel de relevo na epidemiologia do calazar.

As amostras de Leishmania isoladas tanto dos casos humanos, como de casos caninos de LV, caracterizadas bioquimicamente como L. donovani chagasi, apresentaram elevado grau de homogeneidade entre elas; foram, no entanto, distintas das amostras de $L$. braziliensis braziliensis dos casos de LTA de origem humana e canina, isoladas no Rio de Janeiro ${ }^{7}, 14,16$.

Curiosamente, em Campo Grande, na encosta oeste, observou-se um caso humano de infecção concomitante, visceral e tegumentar, correspondente a uma criança de 5 anos que previamente apresentara quadro clínico sugestivo de calazar, veio posteriormente, antes de receber tratamento, a desenvolver lesão cutânea ulcerada (que coincidiu com o aparecimento de lesão do mesmo tipo em uma irmã); a biópsia da lesão cutânea e a punção de medula óssea evidenciaram parasitos caracterizados como L.b. braziliensis e L.d. chagasi, respectivamente ${ }^{15}$.

As tentativas de encontrar animais silvestres, possiveis reservatórios de Leishmania, têm sido infrutíferas; até o momento, 75 roedores de várias espécies e 13 marsupiais foram examinados, sem resultado positivo (dados não publicados).

A distribuição temporal dos casos humanos, segundo a data de diagnóstico, mostrou uma frequeência maior entre os 
meses de maio a agosto. Admitindo-se que o período de incubação, somado ao período de doença anterior ao diagnóstico, seja em torno de 6 meses, é provável que a aquisição da infecção ocorra predominantemente entre os meses de novembro a março, correspondentes aos meses mais quentes do ano, quando a pluviosidade é também maior nas áreas de risco (dados não publicados).

Nas áreas onde a LV humana ou canina foram detectadas, o Ministério da Saúde (SUCAM - Regional RJ) adotou as clássicas medidas de controle: busca ativa e tratamento dos casos humanos, inquérito sorológico canino com eliminação dos cães infectados (assintomáticos ou doentes) e dedetização de todos os domicílios e anexos, repetindo-as semestralmente. Isso reverteu a expectativa de aumento dos casos (Tabela I).

A despeito das inúmeras informações acumuladas, carece ainda de estudos a determinação de fatores ambientais, humanos, sociais, econômicos, etc, que possam ter influenciado a instalação e a propagação da leishmaniose visceral nas áreas peri-urbanas da cidade do Rio de Janeiro.

Sabemos que os desmatamentos no Município remontam ao século XVI, quando da doação das Sesmarias e início de plantações de cana de açúcar. O ciclo cafeeiro, a partir do início do século XIX, induziu à ocupação das encostas do Maciço da Pedra Branca até a cota de $200 \mathrm{~m}$, sendo substituído, no início do século XX, pela fruticultura e implementado, a partir de 1930, com o cultivo dos laranjais, quando os cafezais foram transferidos para o vale do Paraíba. Com a queda do comércio de exportação das frutas cítricas, nos anos que precederam a 2a Guerra Mundial, grandes áreas passaram a ser utilizadas para o plantio de bananais que, galgando progressivamente as encostas, atingiram, em algumas áreas, a altitudes superiores a $500 \mathrm{~m} \mathrm{e}$ assim se mantêm até os dias atuais ${ }^{19}$.

No entanto, a Secretaria Municipal de Planejamento e Coordenação Geral, procurando determinar, com utilização dos dados de sensoriamento remoto por satélite (produtos MSS do Landsat), o ritmo atual de desmatamento, demonstrou que a taxa de crescimento das áreas desmatadas acima da cota 100 , no município, foi de $10,38 \%$ no período de 1972 a 1978; fato esse in terpretado como conseqüência do uso indevido do solo, nos maciços da Pedra Branca, Tijuca e Gericinó, apesar da legislação federal e municipal existente fixar normas de conservação e manutenção de cobertura vegetal nas áreas de encostas acima da cota 100 , ainda remanescentes da Mata Atlântica ${ }^{19}$.

Nesse período, coincidentemente, constatou-se desmatamento longitu dinal, cortando de leste a oeste a reserva florestal do maciço da Pedra Branca - da Estação de Furnas, instalada em Pau da Fome, Jacarepaguá, na vertente leste, até Rio da Prata, Campo Grande, na vertente oeste - para 
a instalação de linhas de transmissão de energia elétrica. Segundo informações, várias companhias empreiteras nacionais participaram dessa obra, através do recrutamento de mão-de-obra de várias procedências, inclusive do nordeste do Brasil, e mantiveram os empregados residindo no local de trabalho em unidades de acampamentos móveis, ao longo de todo o trajeto.

A introdução nessa área do homem ou mesmo do cão, provenientes de outras áreas endêmicas e albergando o parasito, poderia justificar a implantação do mais recente e mais meridional foco de leishmaniose visceral no País associado à presença da Lutzomyia longipalpis e à infecção canina.

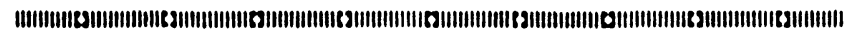

A recente ocorrência de casos humanos e caninos de leishmaniose visceral, autóctones de áreas peri-urbanas da cidade do Rio de Janeiro, a partir de 1977, motivou pesquisadores da Escola Nacional de Saúde Pública e do Instituto Oswaldo Cruz da Fundação Oswaldo Cruz (FIOCRUZ), da Universidade Federal do Rio de Janeiro, da Universidade Federal Rural do Rio de Janeiro, da Secretaria Municipal da Saúde do Rio de Janeiro e da Superintendéncia das Campanhas do Ministério da Saúde (SUCAM-RJ), a participarem de um programa de estudo dessa zooantroponose, visando a uma abordagem ecológica, epidemiológica, parasitológica, imunológica, clinico-terapêutica e de avaliação das medidas de controle adotadas. Resultados gerais dessas pesquisas são apresentados na presente comunicação.

\section{REFERENCIAS BIBLIOGRÁFICAS}

1. ALENCAR, J.E. Leishmaniose visceral no Brasil. $R$. Med. Univ. Fed. Ceará, $17-18$ :129-48, 1978.

2. COUTINHO, S.G. et alii. Anticorpos fluorescentes da classe IgM de imunoglobulinas (IF - IgM) e fator reumatóide na leishmaniose visceral. In: CONGRESSO DA SOCIEDADE BRASILEIRA DE MEDICINA TROPICAL, 18. Ribeirão Preto, 1982. Anais. Ribeirão Preto, 1982.

3. DEANE, L.M. Leishmaniose visceral no Brasil; estudo sobre reservatórios e transmissores realizados no estado do Ceará. Rio de Janeiro, Serviço Nacional de Educação Sanitária, 1956.

4. GALVÃO-CASTRO, B. et alii. Polyclonal B cell activation circulating immune complexes and autoimmu- 
nity in human american visceral leishmaniasis. Clin. Exp. Immunol., 56 : 58-66, 1984.

5. LAINSON, R. et alii. Leishmaniasis in Brazil IV. The fox, Cerdocyon thous (L). As a reservoir of Leishmania donovani In Pará, State Brazil. Trans. R. Soc. Trop. Med. Hyg., 63 : 741-5, 1969.

6. LIMA, L.C.R. et alii. Flebotomíneos em área de ocorrência de leishmaniose tegumentar americana no bairro de Campo Grande, Rio de Janeiro, Brasil. $R$. Bras. Malariol. D. Trop., 33 :64-74, 1981.

7. LOPES, V.G. et alii. Schizodeme and zymodeme characterization of leishmania in the investigation of foci of visceral and cutaneous leishmaniasis. J. Parasitol., 70, 1984.

8. MARZOCHI, K.B.F. et alii. Hemorragia de pele e calazar. In: CONGRESSO DA SOCIEDADE BRASILEIRA DE MEDICINA TROPICAL, 18. Ribeirão Preto, 1982. Anais. Ribeirão Preto, 1982.

9. MARZOCHI, K.B.F. et alii. Hipertensão arterial e calazar. In: CONGRESSO DA SOCIEDADE BRASILEIRA DE MEDICINA TROPICAL, 18. Ribeirão Preto, 1982. Anais. Ribeirão Preto, 1982.

10. MARZOCHI, M.C.A. et alii. Evaluation of diagnostic criteria in human and canine mucocutaneous leishmaniasis in a Rio de Janeiro district where Leishmania braziliensis braziliensis occurs. In: REUNIÃO DE PESQUISA BÁSICA EM DOENÇA DE CHA. GAS, 19. Caxambu, 1982. Anais. Caxambu, 1982.

11. MARZOCHI, M.C.A. et alii. Leishmaniose visceral-calazar. J. bras. Med., $41: 69-84,1981$.

12. MARZOCHI, M.C.A. et alii. Leishmaniose visceral no Rio de Janeiro; aspectos epidemiológicos humanos. In: CONGRESSO DA SOCIEDADE BRASILEIRA DE MEDICINA TROPICAL, 19. Rio de Janeiro, 1983. Anais. Rio de Janeiro, 1983.

13. MARZOCHI, M.C.A. et alii. Leishmaniose visceral no Rio de Janeiro. III - Inquérito epidemiológico pela imunofluorescência e intradermorreação em área de risco de transmissão. In: CONGRESSO DA SOCIEDADE BRASILEIRA DE MEDICINA TROPICAL, 17. Caldas Novas, 1981. Anais. Caldas Novas, 1981.

14. MOMEN, H Identification of new world leishmania isolates by agarose gel eletrophoresis and polyacry. lamide gel soelectrofocusing. J. Cell. Biochem., 70 (Supl. 7A) :29, 1983.

15. OLIVEIRA NETO, M. et alii. Concurrent human infection with Leishmania donovani and $L$. braziliensis 
braziliensis. In: REUNIÃO ANUAL DE PESQUISA BÁSICA EM DOENÇA DE CHAGAS, 19. Caxambu, 1982. Anais. Caxambu, 1982.

16. PACHECO, R.S. et alii. A comparative biochemical analysis of isolates of visceral leishmaniasis from the states of Bahia and Rio de Janeiro. In: JAPAN-BRASIL SYMPOSIUM ON SCIENCE AND TECHNOLOGY, 4. Proceedings. São Paulo, Academia de Ciências, CNPq, 1984. v. 2. p. 58-70.

17. PONTES DE CARVALHO, L. et alii. The pathogenesis on anaemia in american visceral leishmaniasis (AVL). In: INTERNATIONAL CONGRESS OF IMMUNOLOGY, 5. Kyoto, 1983. Abstracts. Kyoto, 1983.

18. RIO DE JANEIRO (cidade). Secretaria Municipal de Planejamento e Coordenação Geral. Diagnóstico do desmatamento nos maciços da Tijuca, Pedra Branca e Gericinó. Rio de Janeiro, 1979.

19. RIO DE JANEIRO (cidade). Secretaria Municipal de Planejamento e Coordenação Geral. Informações básicas da cidade do Rio de Janeiro. Rio de Janeiro, 1979.

20. SABROZA, P.C. O domicilio como fator de risco na leishmaniose tegumentar americana; estudo epidemiológico em Jacarepaguá, município do Rio de Janeiro. Rio de Janeiro, 1981. [Dissertação de Mestrado - Escola Nacional de Saúde Pública, FIOCRUZ]

21. SABROZA, P.C. et alii. Flebotomíneos na cidade do Rio de Janeiro. In: CONGRESSO DA SOCIEDADE BRASILEIRA DE MEDICINA TROPICAL, 14 \& CONGRESSO DA SOCIEDADE BRASILEIRA DE PARASITOLOGIA, 3. João Pessoa, 1978. Anais. João Pessoa, 1978.

22. SOUZA, M.A. et alii. Leishmania visceral no Rio de Janeiro. 1 - Flebotomíneos da área de procedência de caso humano autóctone. Mem. Inst. Oswaldo Cruz, 76 (2) :161-8, 1981

23. TEIXEIRA, R. Experiências vividas com a leishmaniose visceral, 1954-1980; aspectos epidemiológicos, sorológicos e evolutivos. Salvador, 1980. [Tese - Universidade Federal da Bahia]

24. TOLEDO, L.M. et alii. Leishmaniose visçeral na cidade do Rio de Janeiro; casos clínicos do Serviço de Doenças Infecciosas e Parasitárias (DIP) do Hospital Universitário, UFRJ. In: CONGRESSO BRASILEIRO DE MEDICINA TROPICAL, 27. Cal das Novas, 1981. Anais. Caldas Novas, 1981. 
25. TOLEDO, L.M. et alii. Ocorrência de formas assintomá. ticas de leishmaniose visceral humana na localidade de Rio da Prata, Campo Grande, Rio de Janeiro. In: CONGRESSO DA SOCIEDADE BRASILEIRA DE MEDICINA TROPICAL, 19. Rio de Janeiro, 1983. Anais. Rio de Janeiro, 1983. 